\title{
The need for cost-effective choices to treat patients with bipolar 1 disorders including asenapine.
}

\author{
Brian Godman ${ }^{1,2}$ \\ ${ }^{1}$ Department of Laboratory Medicine, Division of Clinical Pharmacology, Karolinska Institutet, \\ Karolinska University Hospital Huddinge, SE-141 86, Stockholm, Sweden. Email: \\ Brian.Godman@ki.se \\ ${ }^{2}$ Strathclyde Institute of Pharmacy and Biomedical Sciences, University of Strathclyde, Glasgow, UK. \\ Email: Brian.godman@strath.ac.uk
}

\begin{abstract}
Bipolar 1 disorders (BPD) are a chronic disorder with prevalence rates up to $2.6 \%$ of the adult population or higher and appreciable direct and indirect costs. As a result, a concern to health authorities especially given the low age of onset. Consequently, a need to treat BPD patients well and improve their quality of life. Pharmacotherapy includes mood stabilisers and atypical antipsychotics (AAPs). AAPs have different mechanisms of action and side-effects so treatment needs to be tailored. Asenapine in clinical trials is as effective as olanzapine with less metabolic side-effects. Chitnis and colleagues have shown in routine care that asenapine also reduces hospital and emergency room admissions making it cost neutral in BPD, which is of interest to health authorities and clinicians.
\end{abstract}

\section{Main Text}

Bipolar I disorders (BPD) are a chronic disorder involving one or more episodes of mania or mixed mood, associated with increased psychomotor activity, excessive social extroversion, decreased need for sleep, impulsivity, impairment in judgment, and grandiose mood. Patients may also experience delusions, paranoid thinking, and extreme agitation $(1,2)$. Published prevalence rates vary from 0.4 to 1.6 percent up to $2.6 \%$ of the adult population $(1,3)$ or higher, especially if the impact of underdiagnosis and misdiagnosis are included $(2,4,5)$. As Chitnis and colleagues point out, in view of the high prevalence of BPD including both psychiatric and associated medical conditions including thyroid disease, migraine, heart disease and diabetes, overall expenditure on this condition is high (3). Direct medical costs were calculated at over US\$30.7billion per year in the US alone in 2009 , with indirect costs greater than US\$120billion annually $(2,3)$.

This high prevalence and costs associated with BPD are a concern to health authorities and health insurance companies world-wide as they struggle with increasing pressures due to ageing populations and the continued launch of new high priced medicines $(6,7)$. Concerns are increased by the low median age of symptom onset at just 20 to 25 years of age $(1,3)$. Consequently, there is a recognised need to treat these patients well to improve their quality of life and reduce emergency room visits and in-patient care.

Pharmacologic treatments for BPD include mood stabilizers, e.g. lithium, valproate, lamotrigine, and carbamazepine, as well as atypical antipsychotics (2). The American Psychiatric Association and others recommend polytherapy, e.g. lithium or valproate in conjunction with an antipsychotic, for patients with severe manic or mixed episodes and monotherapy, e.g. lithium, valproate, or an antipsychotic, for less ill patients with atypical antipsychotics (AAPs) preferred to typical antipsychotics because of their improved side-effect profile (1, 8-10). However other authors have questioned the distinction between first generation (typical) and second generation (atypical) antipsychotics as there are different domains of effectiveness and safety between the different antipsychotics (11). For instance, the extent of extrapyramidal side-effects seen with haloperidol depends on the doses prescribed (11).

Never-the-less, it is recognised by all healthcare professionals that the different AAPs have different mechanisms of action and different effectiveness and safety profiles in patients with major mental illness including BPD $(1,11-14)$. Consequently, the choice of AAP prescribed should be tailored to the individual, especially as there are concerns with the effectiveness of some of the current AAPs for the management of depressive phases of bipolar disorders $(10,13)$. This does not include asenapine, which in short-term trials has demonstrated significant superiority to placebo in treating the manic symptoms of BPD and, in longer term studies, showed comparable efficacy with olanzapine in treating 
manic and depressive symptoms of BPD with less impact than olanzapine on adversely affecting triglycerides, weight and cholesterol levels $(15,16)$. As a result, post-hoc analysis of two short-term clinical trials demonstrated asenapine as a cost-effective alternative to olanzapine in mixed episode BD-I patients, and may have specific advantages in this population, potentially leading to healthcare savings and improved outcomes (17).

However healthcare professionals, especially budget holders, prefer to see the effectiveness, safety and costs of medicines in routine clinical care to aid decision making rather than clinical trials as there can be appreciable differences in patients enrolled into clinical studies versus those seen in routine clinical care, i.e. real life. As a result affecting the generalisability of the findings from clinical trials (7). This is because Phase III clinical trials are typically conducted under ideal and highly controlled conditions to seek high internal validity to maximise the chance of demonstrating clinical benefit (7). Consequently, patients in routine clinical practice may in fact be more elderly and have greater comorbidities than those seen in clinical trials.

This is the strength of the paper by Chitnis and colleagues (3). The authors assessed the impact of asenapine in new users, who are part of two large healthcare claims databases in the US, on subsequent utilisation and health care costs (3). The size of the combined databases, including both commercially insured personnel and Medicare enrolees, is appreciably larger than the total number of patients in most European countries at approximately 30 million. In view of this, providing an appreciable number of patients (1403) meeting the strict entry criteria, which excluded for instance patients with evidence of any use of depot antipsychotics during pre- or post-index as this may indicate patients who have difficulties complying with oral regimes (3). As a result, providing robustness to the findings.

Robustness in the assessment of health care resource utilisation and costs is further facilitated by the comprehensive datasets used and the rigorous validation process. Datasets include detailed in- and out-patient medical claims data, incorporating both diagnoses and procedures, provider types, place of service and total reimbursed costs (3). Pharmacy claims included all medication dispensed and their details including reimbursed amounts.

Encouragingly the authors found that healthcare resources decreased following asenapine. Hospital admissions, emergency room visits, outpatient hospital visits and physicians' office visits were all down (all $p<0.05)(3)$. In addition, the findings were similar among patients with and without evidence of AAPs during the pre-index period. Whilst pharmacy costs increased by on average by US $\$ 839$ per person during the post-index period, this was offset by a corresponding reduction of US\$1806 in the cost of in-patient care, leading to an overall decrease in total BPD related costs of US $\$ 979$. These are total costs lower rather than marginal costs in view of the methodology employed. However, these costs do not include indirect costs, which are substantial for this patient population $(2,3)$.

Whilst the median age of patients with BPD in this dataset was higher at approximately 44 years compared with published studies of at 20 to 25 years of age $(1,3)$, the authors satisfactorily explain this difference. This included the fact that it can take up to 10 years from the time of symptom onset to reach a definitive diagnosis of BPD, and atypical antipsychotics may be reserved until other therapies such as mood stabilisers are found to be ineffective or intolerable (3).

Overall these findings give confidence to all healthcare professionals that asenapine is an effective AAP for this group of patients, reducing inpatient services and emergency room visits, potentially enabling patients to function well enough to benefit from other psychosocial therapies. As mentioned earlier, this meets the goal of health authorities in this area of unmet need. Whilst health authorities typically do not get involved with directing the choice of AAP prescribed in view of the complex issues with managing this population and the need to individualise treatment apart from potentially looking at different dosage forms $(6,18,19)$, despite suggestions to the contrary (13), they should be reassured by these findings. This is different to the situation where there can be concerns with bias in the study findings as seen with for instance with long-acting injectable AAPs where a number of the published studies are open label and subject to significant selection and sponsor bias (20).

We look forward to longer term follow-up of patients prescribed asenapine to give further confidence to all healthcare professionals involved with the management of patients with BPD. 


\section{Acknowledgements}

The author has no conflicts of interest to declare.

\section{Reference}

1. Abou-Setta AM, Mousavi SS, Spooner C, Schouten JR, Pasichnyk D, Armijo-Olivo S, et al. AHRQ Comparative Effectiveness Reviews. First-Generation Versus Second-Generation Antipsychotics in Adults: Comparative Effectiveness. Rockville (MD): Agency for Healthcare Research and Quality (US); 2012. 2. Jann MW. Diagnosis and treatment of bipolar disorders in adults: a review of the evidence on pharmacologic treatments. American health \& drug benefits. 2014;7(9):489-99.

3. Chitnis A, Wang R, Sun SX, Dixit S, Tawah A, Boulanger L. Impact of initiation of asenapine on patterns of utilization and cost of healthcare resources associated with the treatment of bipolar I disorder. Journal of medical economics. 2015:1-23.

4. Bongards EN, Zaman R, Agius M. Can we prevent under-diagnosis and misdiagnosis of bipolar affective disorder? Repeat audits to assess the epidemiological change in the caseload of a community mental health team when bipolar disorder is accurately assessed and diagnosed. Psychiatria Danubina. 2013;25 Suppl 2:S12934.

5. Amihaesei IC. Bipolar disorder, not so rare diagnosis: subtypes of different degrees of severity, diagnosis, therapy. Revista medico-chirurgicala a Societatii de Medici si Naturalisti din lasi. 2014;118(1):111-5. 6. Godman B, Petzold M, Bennett K, Bennie M, Bucsics A, Finlayson AE, et al. Can authorities appreciably enhance the prescribing of oral generic risperidone to conserve resources?: Findings from across Europe and their implications. BMC medicine. 2014;12(1):98.

7. Malmstrom RE, Godman BB, Diogene E, Baumgartel C, Bennie M, Bishop I, et al. Dabigatran - a case history demonstrating the need for comprehensive approaches to optimize the use of new drugs. Frontiers in pharmacology. 2013;4:39.

8. Practice guideline for the treatment of patients with bipolar disorder (revision). The American journal of psychiatry. 2002;159(4 Suppl):1-50.

9. Vieta E, Gunther O, Locklear J, Ekman M, Miltenburger C, Chatterton ML, et al. Effectiveness of psychotropic medications in the maintenance phase of bipolar disorder: a meta-analysis of randomized controlled trials. The international journal of neuropsychopharmacology / official scientific journal of the Collegium Internationale Neuropsychopharmacologicum (CINP). 2011;14(8):1029-49.

10. Yatham LN, Kennedy SH, Parikh SV, Schaffer A, Beaulieu S, Alda M, et al. Canadian Network for Mood and Anxiety Treatments (CANMAT) and International Society for Bipolar Disorders (ISBD) collaborative update of CANMAT guidelines for the management of patients with bipolar disorder: update 2013. Bipolar disorders. 2013;15(1):1-44.

11. Leucht S, Cipriani A, Spineli L, Mavridis D, Orey D, Richter F, et al. Comparative efficacy and tolerability of 15 antipsychotic drugs in schizophrenia: a multiple-treatments meta-analysis. Lancet. 2013;382(9896):951-62. 12. Selle V, Schalkwijk S, Vazquez GH, Baldessarini RJ. Treatments for acute bipolar depression: metaanalyses of placebo-controlled, monotherapy trials of anticonvulsants, lithium and antipsychotics. Pharmacopsychiatry. 2014;47(2):43-52.

13. Singh AB, Nierenberg AA, Yatham LN, Berk M. Atypical antipsychotic agents; Peas in a pod or chalk and cheese? BMC medicine. 2014;12:126.

14. Cerullo MA, Strakowski SM. A systematic review of the evidence for the treatment of acute depression in bipolar I disorder. CNS spectrums. 2013;18(4):199-208.

15. Vita A, De Peri L, Siracusano A, Sacchetti E. Efficacy and tolerability of asenapine for acute mania in bipolar I disorder: meta-analyses of randomized-controlled trials. International clinical psychopharmacology. 2013;28(5):219-27.

16. Kemp DE, Zhao J, Cazorla P, Landbloom RP, Mackle M, Snow-Adami L, et al. Weight change and metabolic effects of asenapine in patients with schizophrenia and bipolar disorder. The Journal of clinical psychiatry. 2014;75(3):238-45.

17. Sawyer L, Azorin JM, Chang S, Rinciog C, Guiraud-Diawara A, Marre C, et al. Cost-effectiveness of asenapine in the treatment of bipolar I disorder patients with mixed episodes. Journal of medical economics. 2014;17(7):508-19.

18. Parks J, Radke A, Parker G, Foti ME, Eilers R, Diamond M, et al. Principles of antipsychotic prescribing for policy makers, circa 2008. Translating knowledge to promote individualized treatment. Schizophrenia bulletin. 2009;35(5):931-6.

19. Godman B, De Bruyn K, Miranda J, Raschi E, Bennie M, Barbui C, et al. Generic atypical antipsychotic drugs in Belgium: their influence and implications. Journal of comparative effectiveness research. 2013;2(6):55161.

20. Chue $P$, Chue J. The cost-effectiveness of risperidone long-acting injection in the treatment of schizophrenia. Expert review of pharmacoeconomics \& outcomes research. 2012;12(3):259-69. 